\title{
Case Series of Irritated Giant Acrochordons Arising from Skin Folds
}

\author{
Fiedyawati Kusuma**iD, Jemie Rudyan² (D) \\ ${ }^{1}$ Department of Dermatology, Venereology, Mulya Hospital, Ciledug, Tangerang, Indonesia; ${ }^{2}$ Department of Orthopaedics and \\ Traumatology, Faculty of Medicine, Udayana University, Sanglah General Hospital, Denpasar, Bali, Indonesia
}

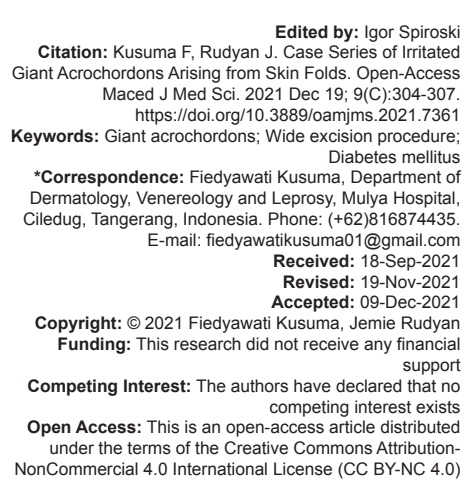

Introduction

Acrochordons are pedunculated papules or nodules with a soft consistency and smooth contour, sometimes apparently growths often hang on thin stalk, and the most acrochordons size was 2-5 mm. Acrochordons usually have incidence of $46 \%$ in female reproductive age group, and may relate to the growth hormone like activity of insulin. Hence, they may associate with diabetes mellitus. Occasionally, as a result of twisting or frictions of the pedicle one will become inflamed, tender, and even gangrenous [1], [2], [3], [4].

Acrochordons are also referred to as skin tags or fibroepithelial polyps in size more than $5 \mathrm{~cm}$ are rare, especially when located in vulva [5]. They typically develop from skin of the eye lids, neck, axilla, groins, upper thighs, and trunk, but they can emerge from any part of the body [1], [2], [3], [4], [6]. Acrochordons do not appear to be infectious or malignant by themselves [1], [5].

Management depends on the size of the acrochordons: From simple clipped/shave off, light electrodesiccation, and complete excision and cosmetic repair for bigger size acrochordons [1], [2], [4], [5].

\section{Case Presentation 1}

A 19-year-old female come to my OPD clinic with a big mass on her left vagina, started as a small bump around 2 years ago; however, it progresses in size, with no other symptoms. Eventually, it becomes very big which apparently causing her straddle position while walking, particularly a week before clinic, it caused a very pain and hot while being touched, and fever.

\section{On examination}

There was a big nodule on the right labia mayor, diameter $20 \times 20 \mathrm{~cm}$, short stalk, firm, warmed, and pain sensation while touched, with several excoriated skin surface. No increase in size of the mass with valsalva maneuver (Figures 1 and 2). 


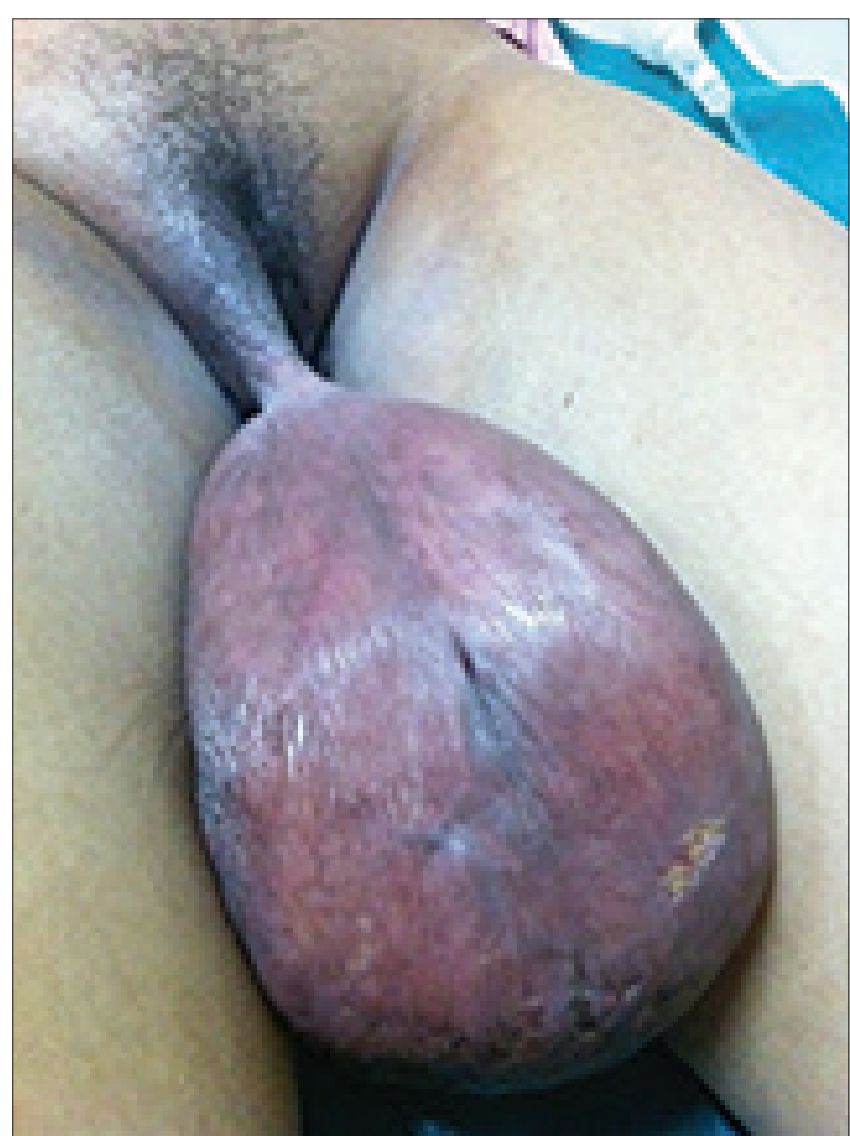

Figure 1: Giant acrochordons before surgery

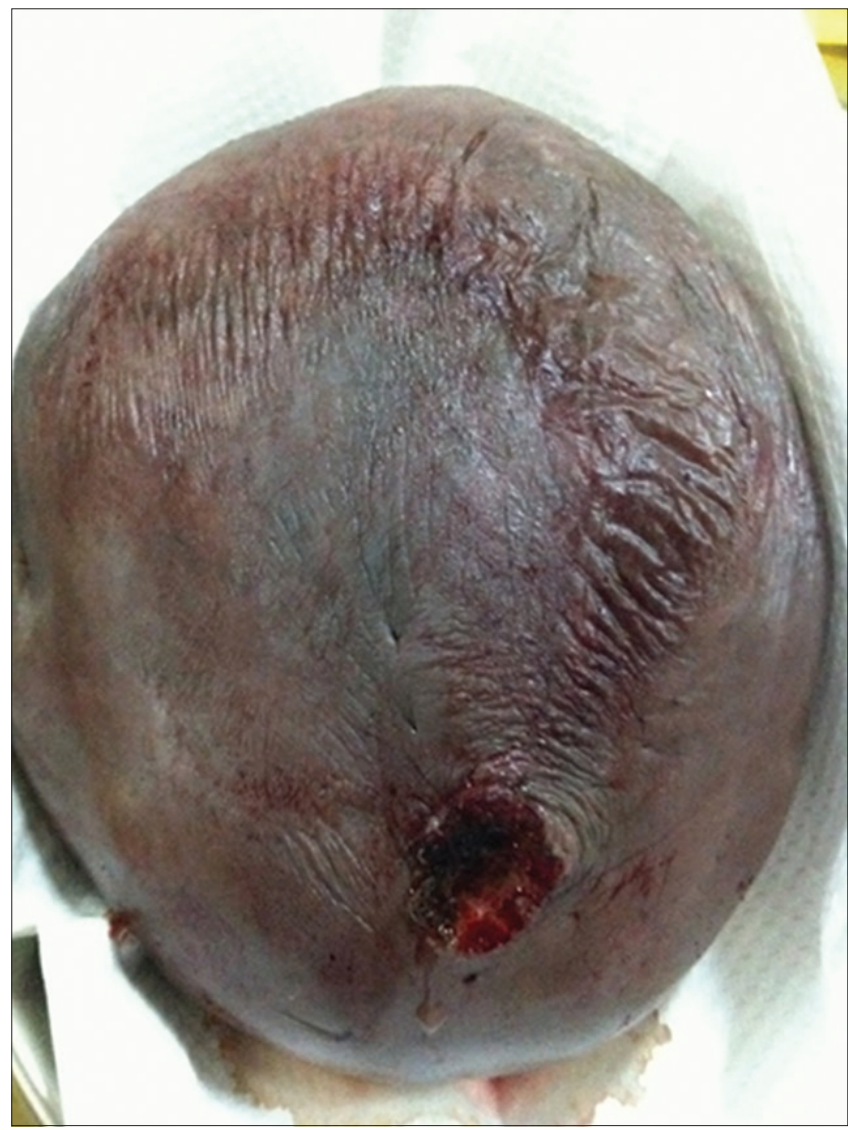

Figure 2: Giant acrochordons after surgery

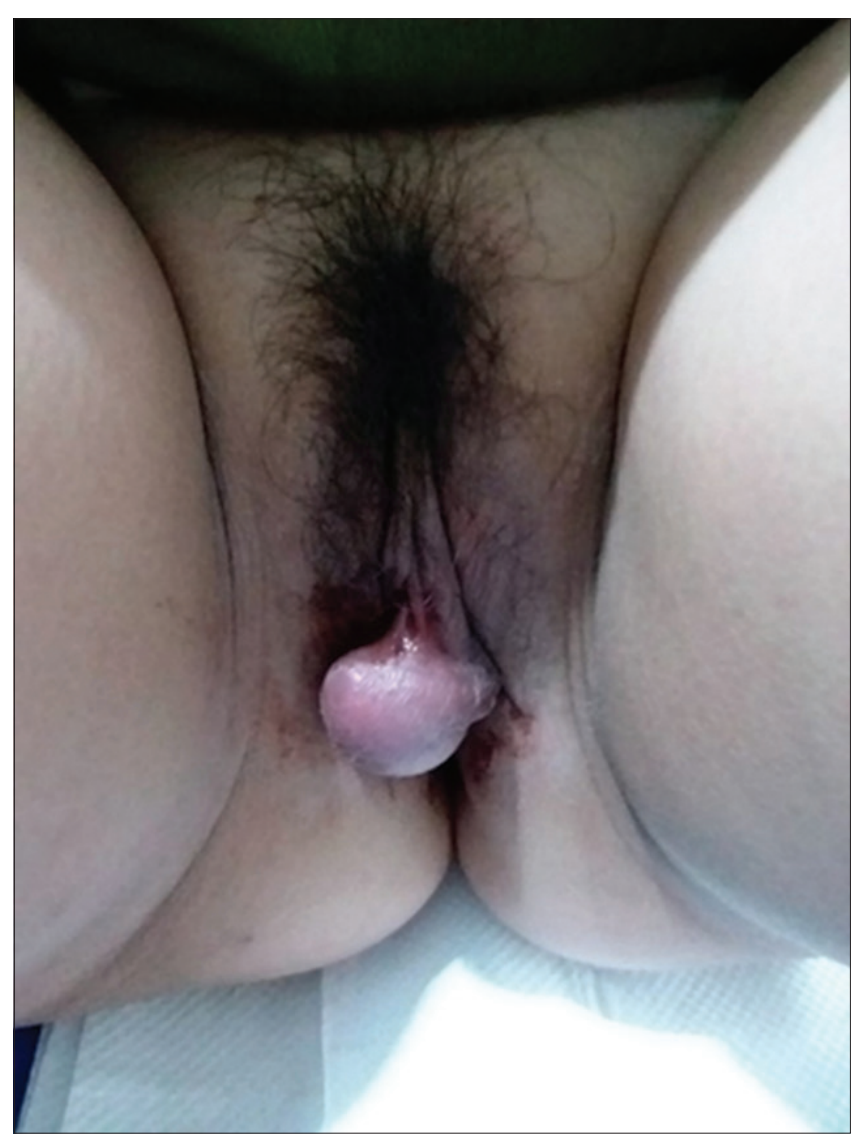

Figure 3: Giant acrochordons before surgery

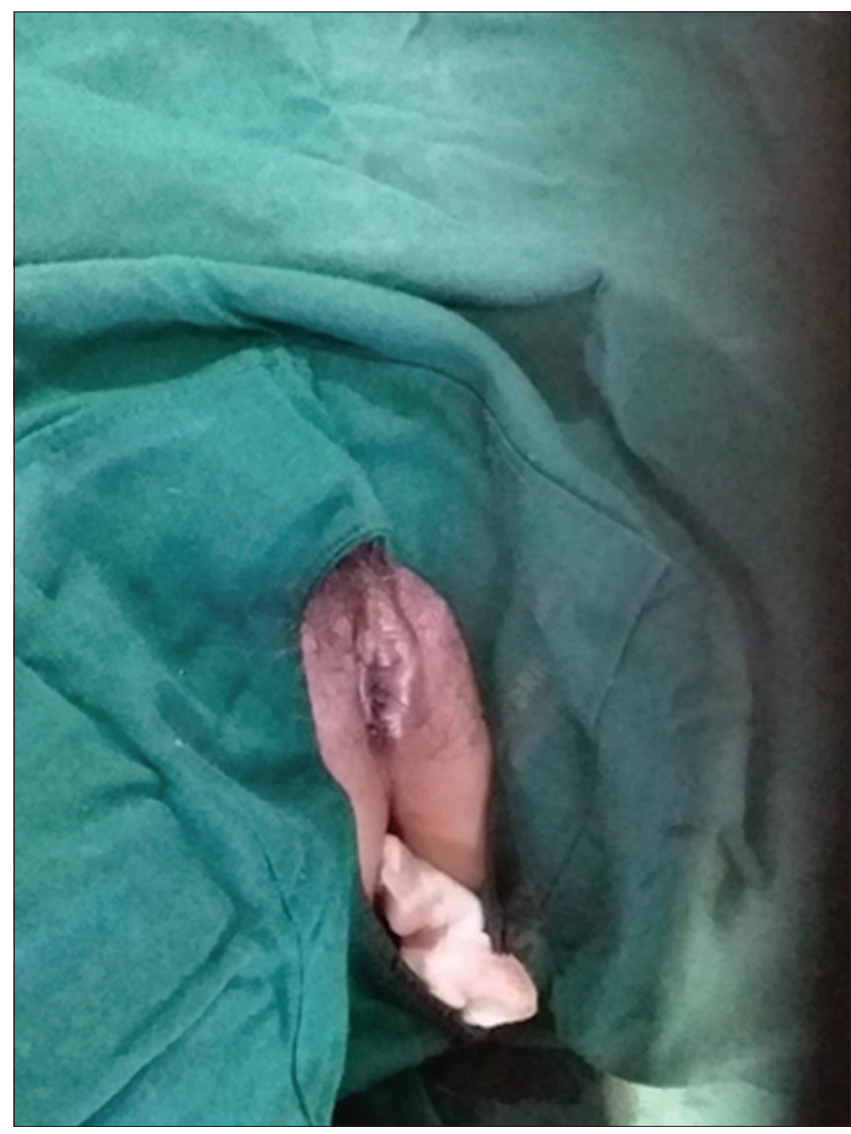

Figure 4: Giant acrochordons after surgery 


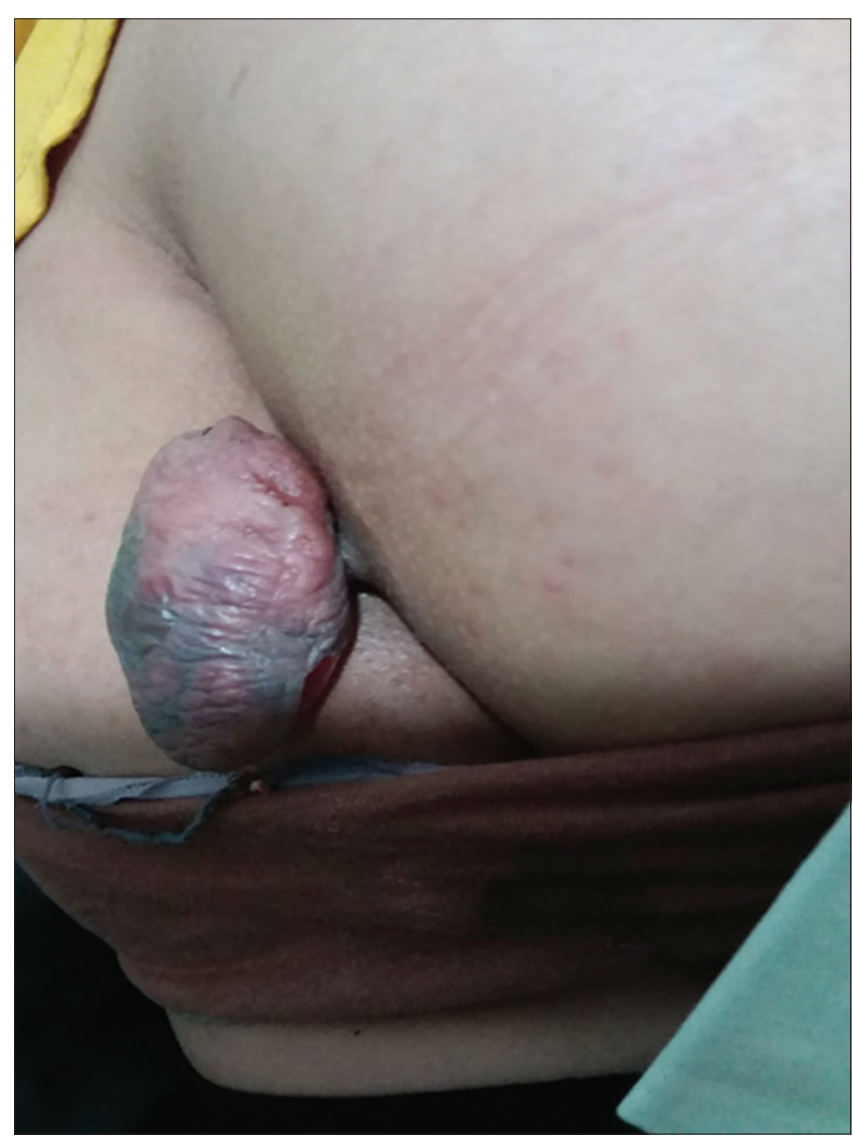

Figure 5: Giant acrochordons before surgery

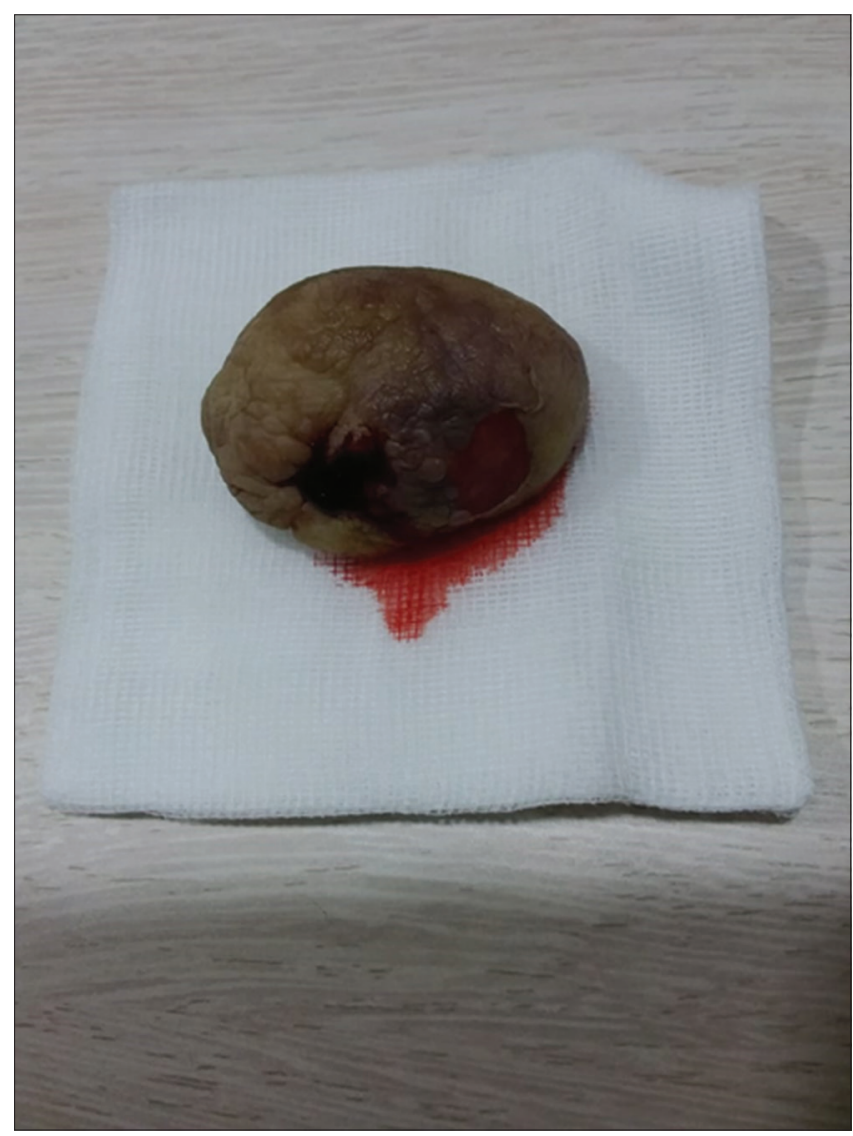

Figure 6: Giant acrochordons after surgery
We performed USG examination and found multiple lymph nodes on the right inguinal with various sizes $0.81-0.85 \times 1.0 \mathrm{~cm}$, no sign of inguinal hernia. On complete blood examination, leukocytosis was found; other findings appear normal.

Pathology anatomy finding shows epilated squama and papillomatosis structure, with edema, stellate stroma, and proliferative blood vessels, no mitosis was found. Final assessment of the patient is angiomyxoma.

\section{Case Presentation 2}

A 23-year-old female presented in my OPD with a mass in her left vagina, which started in her childhood; however, it increasing in size, particularly after having menstrual periods. Eventually, it become very big in size, associated with pain and several bleeding spots while being touched.

\section{On examination}

Firm solitary erythematous nodule from the left labia mayor, with blood spotting and excoriated skin. Nodule with diameter of $5 \times 5 \mathrm{~cm}$, is also accompanied with pain and hot sensation. We then assess the patient as irritated giant acrochordon, and then performed wide excision of the lesion under local anesthesia. Pathology anatomy slide showing polyploidy epilated flat epithelial, stroma showing chronic inflammations, and necrosis area was found together with acute and chronic inflammation cells. Assessment: Histologic features showing fibroepithelial papilloma and abscess, no sign of atypical and malignancy (Figures 3 and 4).

\section{Case Presentation 3}

A 31-year-old female consulted to OPD clinic with a year of history having a mass on her right buttock, which gradually increases in size and causing a light pain particularly while sitting with spots of bleeding.

\section{On examination}

The right buttock with a short pedunculated nodule, diameter $10 \times 6 \mathrm{~cm}$, oval shape, firm, several excoriated plaques, and several hematoma formations. We assess the patient as irritated giant acrochordons and performed electrodesiccation and curettage (Figures 5 and 6). 


\section{Discussion}

The previous studies state that the larger lesions of acrochordons in the groin or upper thighs maybe associated with diabetes mellitus and obese individuals [1], [2], [3], [4], [7]. However, in the three of these cases had no sign of insulin resistance, instead they were in their normal proportional body weight.

It was mentioned as a result of twisting or strangulated of the pedicle, it will become inflamed, tender, irritated, infarction, and even gangrenous [1], [2], [5], [6], [8]. The three of these cases showing irritated due to the frictions and pressure to the skin surface, hence they become reddish, pain, and excoriated wounds on those mass.

The first case is a neglected case, since patient is a young female, whom stays in an Islamic boarding school. She did not dare to mention that big mass to anybody due to fear and shy feeling for having that. It was discovered when she went back home and has a peculiar walking style with straddle position and pain grimace facial expression, together with having high body temperatures.

The second case before coming to dermatology department had history of having consultation with an obstetrician, which was then diagnosed as Bartholin cyst and questioned about history of sexual intercourse. This perhaps caused by the short of the stalk which almost seen part of the mass [4]. Eventually, she searched for the second opinion. The mass had caused the drooping of the left labia mayor, so it is looked oversized compared to the right size. During the procedure, I had chosen a wide technique excision for removing of the nodule and repairing of the uneven sized of the labia mayor.

The third case was also be differentiated with infantile perianal pyramidal protrusions which mostly occur in young children, usually girls, in the mid line anterior to the anus which reduces with time and no treatment is necessary [2]. However, in this case, the stalk was appeared not sharply from the mid line of the body surface.

It was advised to have the acrochordons removed, particularly in the skin fold of axillae, groin, or vagina, since they will gradually develop in size, perhaps due to the increasing of blood supply which manifested by frictions, pressures activities, and particularly during pregnancy which may relate to the growth hormone like activity of insulin [2], [3], [4], [7], [8], [9].

\section{Conclusion}

Giant acrochordons usually relate to the growth hormone like activity. Hence, they may associate with endocrine disorder such as diabetes mellitus. Giant acrochordons may occasionally become inflamed, tender, and even gangrenous due to frictions of the pedicle. Although management of giant acrochordons may vary, the general principle is to have the acrochordons removed, particularly in skin fold area.

\section{References}

1. Fitzpatrick TB, Wolff K, Goldsmith LA, Katz SI, Gilchrest BA Paller A, et al. Fitzpatrick's Dermatology in General Medicine. New York: McGraw-Hill Medical; 2008.

2. Parish LC. Andrews' diseases of the skin: Clinical dermatology. JAMA. 2011;306(2):127. https://doi.org/10.1001/ jama.2011.968

3. Alkhalili E, Prapasiri S, Russell J. Giant acrochordon of the axilla. BMJ Case Rep. 2015;2015:bcr2015210623. https://doi. org/10.1136/bcr-2015-21062 PMid:26142392

4. Garg S, Baveja S. Giant acrochordon of labia majora An uncommon manifestation of a common disease. J Cutan Aesthet Surg. 2015;8(2):119-20. https://doi. org/10.4103/0974-2077.158454

PMid:26157316

5. Agrawal A, Garg C, Mukherjee S, et al. Giant acrochordon of vulva: A rare occurrence. Nepal J Dermatol Venereol Leprol. 2015;13(1):70-2. https://doi.org/10.3126/njdvl.v13i1.14310

6. Sancaktutar AA. An unusual mass on the skin of scrotum Acrochordon. J Clin Anal Med. 2015;6(1):112-3. https://doi. org/10.4328/jcam.794

7. Can B. Giant fibroepithelial polyps: Why do they grow excessively? SiSli Etfal Hastan Tip Bul. 2020;54(2):257-60. https://doi.org/10.14744/semb.2018.33603 PMid:32617069

8. Özbey R, Türkmen D. A rare case: A giant acrochordon and skin ulcer due to the traction of acrochordon. Turk Klin Dermatol. 2020;30(2):63-5. https://doi.org/10.5336/ DERMATO.2019-65660

9. Galeana C, Casas D, Rodríguez A, Cantú MA, Aguilar F, Moreno $\mathrm{V}$, et al. Benign tumors of vulva: Review and case report of achrocordon. Medwave. 2014;14(1):e5886. https://doi. org/10.5867/medwave.2014.01.5886

PMid:25191903 